\title{
A multiscale method for the analysis of defect behavior in Mo during electron irradiation
}

\author{
J. Rest, Z. Insepov, B. Ye, D. Yun \\ Argonne National Laboratory, 9700 S. Cass Avenue, Lemont, IL 60439, USA, \\ Jeff.Rest@gmail.com
}

Submitted for publication in Computational Materials Science

Revised, April 2014

Argonne National Laboratory's work was supported by the U.S. Department of Energy, Office of Nuclear Energy, under contract DE-AC02-06CH11357.

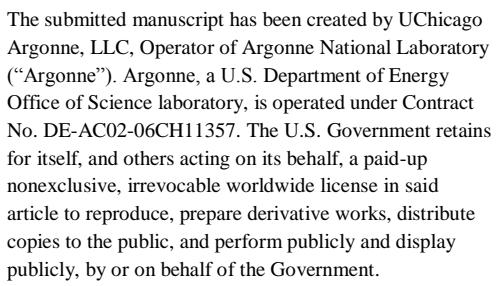

(C) 2014. This manuscript version is made available under the Elsevier user license 


\title{
A multiscale method for the analysis of defect behavior in Mo during electron irradiation
}

J. Rest, Z. Insepov, B. Ye, D. Yun

Argonne National Laboratory, 9700 S. Cass Ave., Argonne, IL 60439, USA

\begin{abstract}
In order to overcome a lack of experimental information on values for key materials properties and kinetic coefficients, a multiscale modeling approach is applied to defect behavior in irradiated Mo where key materials properties, such as point defect (vacancy and interstitial) migration enthalpies as well as kinetic factors such as dimer formation, defect recombination, and self interstitial-interstitial loop interaction coefficients, are obtained by molecular dynamics calculations and implemented into rate-theory simulations of defect behavior. The multiscale methodology is validated against interstitial loop growth data obtained from electron irradiation of pure Mo. It is shown that the observed linear behavior of the loop diameter vs. the square root of irradiation time is a direct consequence of the 1D migration of self-interstitial atoms.
\end{abstract}

\section{Introduction}

Developing a predictive model for analyzing the irradiation behavior of materials has been plagued by the lack of reliable values for materials properties and the difficulty in performing separate-effects studies in extreme environments that are, by their very nature, multivariate. This situation has been compounded by the severe decrease in basic experimentation on irradiated nuclear materials since the 1970 s.

In general, the irradiation behavior of nuclear materials has been modeled by using rate theory formulations $[1,2]$. In this approach, the gain and loss of irradiation-generated defects and impurities are tracked by various terms in the constitutive equations, each term being associated with an assumed behavioral mechanism. The analytic forms of the terms representing the various physical processes are generally derived based on a continuum approach from mean field theory; they are defocused from the specific details and variations present at the atomistic level. Hence, questions arise about the validity of such expressions for realistically describing the phenomena. Put another way, what order of uncertainty is associated with each expression that describes a specific behavioral mechanism?

The situation is compounded by the lack of experimental information on values for key materials properties: for example, diffusion coefficients and nucleation rates. In general, these properties are "predicted" with a rate-theory approach by adjusting them to obtain the best overall agreement with the available data. However, the uncertainties in these properties and parameters generate uncertainty in the validity of the underlying physics and in the physical reality of proposed behavioral mechanisms. This uncertainty clouds the predictive aspects of any mechanistic approach to describing the phenomena. Thus, more detailed data is required in order to help clarify these issues. In what follows, MD simulations are used in order to provide this data. 
The multiscale methodology described in this paper consists of three primary components: (1) a higher length- and time-scale component suitable for simulating in-reactor and electron and ion beam irradiations, namely, a rate theory; (2) a lower time- and length-scale component, for example, molecular dynamics (MD) for an atomistic calculation of key materials properties, such as point-defect (vacancy and interstitial) migration enthalpies, as well as kinetic factors, such as dimer formation, defect recombination coefficients, and self-interstitial atom-interstitial loop reaction rates that are required by the larger length scale calculation; and (3) validation of the combined higher and lower time- and length-scale calculations against in-reactor and/or electron and ion-beam irradiation data.

MD cannot extend the results of concentration calculations to the experimental time scale for irradiated materials. Therefore, MD is employed to calculate kinetic coefficients that are then combined with the rate theory. As such, in order to demonstrate the viability of the methodology, a rate-theory model is utilized in an exercise to interpret the evolution of dislocation loops in irradiated molybdenum. Calculations of the dose dependence of average loop density and diameter were performed and compared with experimental measurements obtained from irradiations with 1-2 MeV electrons. These experiments were selected based on their relative simplicity: electron irradiation where damage is by Frenkel pair production and cascade effects are minimal; and the simulation of experiments that employ pure Mo, thus eliminating impurity effects; it is through the process of implementing such a multiscale approach of combining molecular dynamics and rate-theory modeling that a predictive performance model of materials behavior in irradiation environments can be established.

In a previous paper [3], basic properties (such as formation energies and structure) of self-interstitial atoms (SIA's) in Mo were studied using density functional theory (DFT) $[4,5]$. It was found that the difference in formation energies of $\langle 111\rangle$ crowdion and dumbbell configurations is negligibly small, which implies high SIA mobility along the $\langle 111\rangle$ direction at low temperatures in agreement with resistivity recovery experiments [6]. Empirical interatomic potentials for Mo [7, 8] are not capable of interpreting the hierarchy of SIA formation energies and do not predict one-dimensional motion of SIA's (e.g., as is demonstrated in this work). As a consequence, the use of these potentials results in a relatively slow SIA diffusion at low temperatures. A recently proposed potential [9] correctly reflects the hierarchy of the defects, but predicts an anomalously large thermal expansion, and also overestimates the Mo melting temperature.

\section{Atomistic Simulations}

A new Mo interatomic Embedded Atom Method (EAM) potential has been developed [3] by means of a force matching method [10- 12] that correctly describes thermodynamic properties and energies of the main defect structures in Mo. The main idea of the method is fitting the potential to $a b$ initio atomic forces for a set of atomic configurations of different structures. The interatomic potential was constructed by generating a dataset of atomic forces with densityfunctional calculations performed with the VASP code [10,11], taking into account a number of structures with vacancies and interstitials of several types. This method reproduces relative energies of the defects in accordance with the $a b$ initio calculations, which is important for a physically correct description of defect migration $[9,13]$. 
The force-matching method $[11,12]$ was used for the development of the potential as implemented in the POTFIT code. [12] This method provides a way to construct physically justified interatomic potentials excluding the experimental data from the fitting database. The idea is to adjust the interatomic potential functions to optimally reproduce per-atom forces (together with total energies and stresses) computed at the ab initio level for a fine-tuned set of reference structures.

The reference data were calculated using the DFT code VASP [13] with approximately 200 atoms in a super-cell (the number of atoms depends on the structure, the number of defects, etc.). Projector augmented-wave (PAW) pseudo-potentials together with the plane-wave basis cutoff energy of $400 \mathrm{eV}$ were used. The Brillouin zone was sampled with the $2 \times 2 \times 2$ Monkhorst-Pack k-point mesh. The generalized gradient approximation (GGA) of Perdew and Wang [15] was used for the exchange-correlation functional.

81 configurations with a total of 10,746 atoms were used. These configurations represent 39 states with pure Mo (liquid and bcc solid at different densities; lattice with SIAs and/or vacancies and/or surface), 20 states with pure Xe (liquid and solid), and 22 states with Mo and Xe (including a single Xe atom in pure Mo). The energies and stresses for different configurations of point defects were included into the target function with higher weights. Initially, all the atomic configurations were taken from classical MD runs at different temperatures performed with some preliminary EAM potential. The fitting procedure consists of the following steps: (1) fitting the parameters of the potential to the ab initio database, (2) testing the potential with respect to certain properties, and (3) recalculating the initial set of configurations with the updated potential. This procedure is performed in an iterative manner in order to improve the description of the desired properties.

The developed potential reproduces energies, stresses, and per-atom forces of the reference data with the average accuracy $0.5 \%, 4 \%$, and $25 \%$, respectively. For simulations of high-energy collisions, the pair parts of the EAM potential are modified at small distances to transform smoothly into the universal screened Coulomb potential. [16] In what follows this potential will be referred to as the Argonne Potential.

The basic properties of pure molybdenum calculated with the Argonne potential and two previously published EAM potentials are summarized in Table I and compared with the experimental data. The accuracy of the Argonne potential is slightly lower than that of its predecessors at room temperature and zero pressure. However, the Argonne potential has significant advantage in the description of processes over a wide range of pressures and temperatures [4]. For example, the Argonne EAM potential reproduces the room-temperature isotherms of Mo and Xe up to approximately $600 \mathrm{GPa}$, in agreement with experimental data [1720]. The Argonne potential also reproduces the isothermal compression of pure Mo with good precision. Thermal expansion at zero pressure is well reproduced [18] up to the melting point with $1 \%-2 \%$ accuracy.

The melting of pure molybdenum was also investigated using two-phase simulation. [2123]. The calculated melting line of pure Mo is very close to the DFT results of Ref. 24, and agrees well with the shock-wave test data [25]. 
TABLE I. Comparison of EAM interatomic potentials for molybdenum at room temperature for calculated cohesive energy $E_{c}$, equilibrium lattice parameter a, elastic constants $C_{i j}$, and melting temperature $\mathrm{T}_{\mathrm{m}}$. The experimental data are taken from Refs. 17-19.

\begin{tabular}{|l|l|l|l|l|}
\hline & Experiment & EAM (Ref. 9) & EAM (Ref. 10) & $\begin{array}{l}\text { EAM (this } \\
\text { work) }\end{array}$ \\
\hline Ec $(\mathrm{eV})$ & 6.82 & 6.85 & 6.84 & 6.88 \\
\hline $\mathrm{a}(\AA)$ & 3.1472 & 3.1471 & 3.1465 & 3.1470 \\
\hline $\mathrm{C} 11(\mathrm{GPa})$ & 465 & 420 & 600 & 550 \\
\hline $\mathrm{C} 12(\mathrm{GPa})$ & 176 & 170 & 169 & 220 \\
\hline Tm $(\mathrm{K})$ & 2890 & & 3350 & 2630 \\
\hline
\end{tabular}

The Argonne potential reproduces the cold curve in agreement with the experimental data up to approximately $600 \mathrm{GPa}$ (corresponding compression V/V0 0.5) which gives improved accuracy for the description of heavily irradiated materials at high fluence as compared to existing potentials, including a recent potential that was developed using DFT [10]. In addition, the description of thermal expansion is well replicated up to the melting point. In the present paper, this new Argonne potential is applied to the study of irradiation produced defects in Mo.

A consequence of the new Argonne potential is that the most stable configurations of interstitial defects are $<111>$ dumbbell and $<111>$ crowdion with very small differences in formation energies, and are thus constantly converting from one type to another by overcoming a small energy barrier [14]. This is in contrast to results obtained using empirical potentials where the most stable configurations are along the $\langle 110\rangle$ direction. A dumbbell defect formed along a $<110>$ direction (a rotation form) has a larger energy [13], and exists for only a short time. These $<111>$ configurations also provide conditions for fast 1D migration of self-interstitial atoms (SIAs) even at a very low temperature [6], in good agreement with the resistivity recovery measurements conducted after electron irradiation [3].

With an increase in temperature, <111> dumbbells can also be transformed into a $<110>$ dumbbell state with a small activation energy of rotation, which provides a viable mechanism for switching from 1D motion at low temperature to 3D diffusion at high temperature.

The formation energies of the crowdion versus dumbbell orientation in Mo obtained by molecular statics calculations using the Argonne EAM potential are presented in Figure 1. The $<111>$ dumbbell and crowdion defects are clearly stable in Mo. However, the difference between the crowdion and dumbbell energies in Mo is extremely small near the $\langle 111\rangle$ orientation. This means that a $<111>$ dumbbell is thermally indistinguishable from the crowdion in the $<111>$ direction. The results shown in Figure 1 obtained by molecular statics calculations using the new EAM potential (Argonne potential) are in good agreement with the DFT data of Han et al. [4].

A typical size of a simulation cell for the calculation of SIA and SIA reactions was taken as $100 \times 100 \times 100$ lattice units $(31 \times 31 \times 31 \mathrm{~nm})$. The simulated system contained of about 2 million 
atoms. The initial numbers of crowdions were 300, 400, and 600. For evaluation of vacancy and SIA recombination rates, a $100 \times 100 \times 100$ system with 200 crowdions and 800, 1000, 1500, and 2000 vacancies was used, and a $150 \times 150 \times 150$ system with 200 crowdions and 3000, 4000, 5000, and 6000 vacancies was applied. During the calculation the vacancies were assumed to be immobile. The small number of crowdions was used in order to exclude the formation of SIA clusters and to elucidate the recombination kinetics. In all cases, 3D periodic boundary conditions were used. The centro-symmetry and the potential energy per atoms parameters were used as local variables in order to characterize the symmetry of the local environment. The variable obtained by a common-neighbor analysis was used as a third local order parameter in order to characterize the symmetry of the lattice: $h c p, b c c, f c c$. All calculations were conducted at room temperature.

The rate equations describe defect evolution during the kinetic phase of the irradiation damage spectrum (i.e. as compared to the ballistic phase). The MD simulations therefore focus on the diffusion-based relatively long time evolution of the defects and bypass the computationally expensive ballistic phase. This scenario resembles the situation at the end of the ballistic phase of the irradiation damage simulation. By using this method, the key kinetic coefficients required by the rate theory formulation can be extracted.

Three different types of initial defect configurations were considered: a system containing only SIA existing as crowdions, a system with both crowdions and vacancies, and a system containing crowdions and dislocation loops with Burgers vector in the $\langle 111\rangle$ direction. This set of initial defect configurations enables a separate kinetic study of loop formation via SIA association and/or SIA recombination with vacancies and loops.

The initial spatial distribution of defects was assumed to be uniform for both crowdions and vacancies. The crowdions have the four equivalent directions in the basic crystal cell: [111], [ $\overline{1} 11],[1 \overline{1} 1]$, and [11 $\overline{1}]$. It was assumed that the equivalent orientations were also uniformly distributed.

The evolution of the microstructure of Mo experiencing radiation damage was investigated from the initial state to the point at which the annealing of defects becomes viable. The point defects in an ideal bcc Mo crystalline structure were introduced into the system at the beginning of the simulation, and defect evolution was subsequently studied by following the defect kinetics. In order to simplify the analysis and the kinetics, the damage rate during the calculations was set to zero.

Two types of SIA dimers (diSIAs) are stable: one mobile and the other immobile. Here a dimer is an association of two parallel $\langle 111\rangle$ crowdions/dumbbells moving in concert (mobile configuration), or an association of crossed $<111\rangle$ crowdions/dumbells (immobile configuration). Kinetic rate equations were written to correspond to the particular set of defect configurations used in the MD simulation and were solved analytically. 


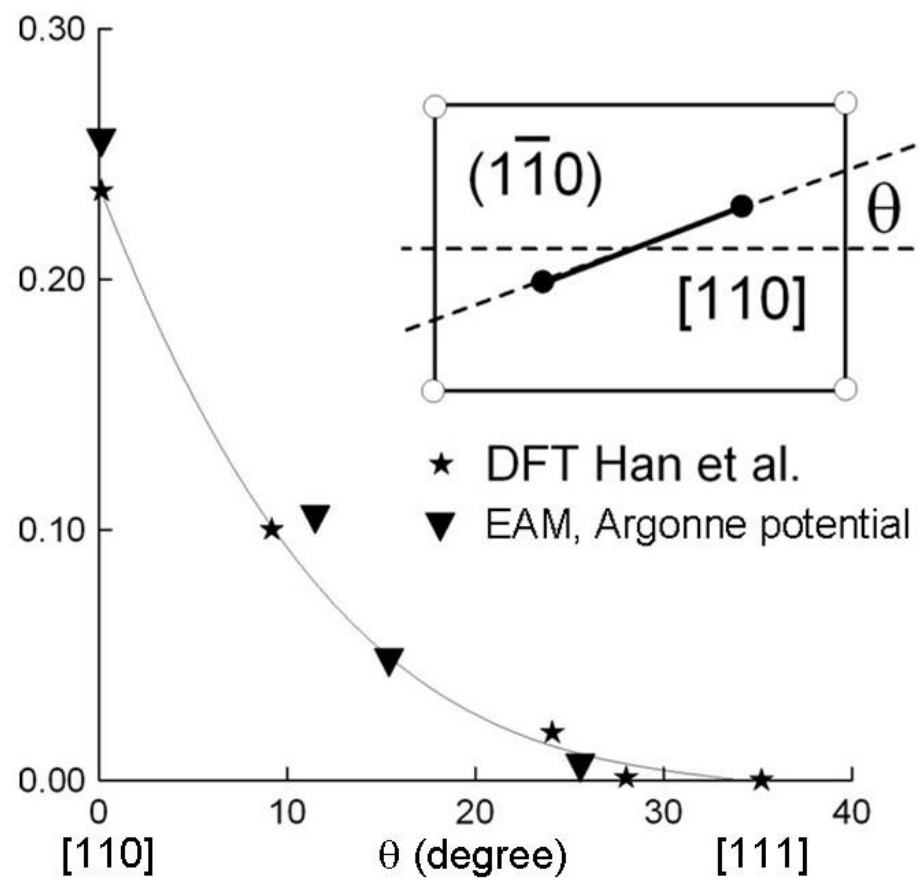

Figure 1. The formation energy of the self-interstitials in Mo (vertical axis), with orientations between $\langle 111\rangle$ and $\langle 110\rangle$ relative to that for the $\langle 111\rangle$ dumbbell, calculated using an ab initio DFT method [4] compared to results obtained using the new Argonne EAM potential [3]. $\theta=0$ corresponds to the [110] axis and $\theta=\tan ^{-1}(1 / \sqrt{2}) \approx 35.3^{\circ}$ is along the [111] axis.

\section{Kinetic rate theory simulations}

The standard kinetic equations for defect behavior in irradiated Mo are described as follows [26]:

$$
\begin{aligned}
& \frac{d c_{i}}{d t}=K \quad{ }_{i v} c_{i} c_{v} \quad{ }_{i i} c_{i} c_{i} \quad k_{i} D_{i} c_{i} \quad{ }_{i L} c_{i} N_{L}, \\
& \frac{d c_{v}}{d t}=K \quad{ }_{i v} c_{i} c_{v} \quad k_{v} D_{v} c_{v} \quad{ }_{v L} c_{v} N_{L}, \\
& \frac{d N_{l}}{d t}=\frac{1}{2}{ }_{i i} c_{i} c_{i} /, \\
& \frac{d m_{L}}{d t}={ }_{i L} c_{i} \quad{ }_{v L} c_{v},
\end{aligned}
$$

where $K$ is the damage rate; $c_{i}, c_{v}, N_{L}$ are the average SIA, vacancy, and interstitial loop density, respectively; $m_{L}$ is the average number of atoms in the average-size loop; $k_{i}$ and $k_{v}$ are the sink strengths for SIA's and vacancies, respectively; $D_{i}$ and $D_{v}$ are the interstitial and vacancy diffusivities; and ${ }_{i v},{ }_{i i},{ }_{i L}$, and ${ }_{v L}$ are the kinetic coefficients (rate constants) in terms of binary reactions. The equations were further simplified by investigating limiting 
conditions and solved analytically. The analytical solutions are compared with MD results for the same limiting conditions and the kinetic coefficients are thus obtained. Fast parallel calculations were carried out on the "Fusion" supercomputer at Argonne National Laboratory by using the LAMMPS molecular dynamics simulation package [15].

\section{Calculation of Diffusion and Kinetic Coefficients by Molecular Dynamics}

The diffusion coefficients of SIA and vacancies were calculated by relating the accumulation of stochastic displacement of a particle in a time unit with the microscopic diffusion coefficient, which can be calculated by using large-scale MD simulations:

$D=1 / 6 \lim _{t \rightarrow \infty} d\left\langle|\vec{r}(t)-\vec{r}(0)|^{2}\right\rangle / d t$

where $r(0), r(t)$ are the radius vectors of the particles at two-time instants, and $D$ is the diffusion coefficient. The results for SIA diffusion are as follows: $D_{i}=D_{0} \exp \left(E_{i m} / k T\right)$, where $D_{0}=$ $7.18 \times 10^{-4} \mathrm{~cm}^{2} / \mathrm{s}$ and $E_{i m}=0.02 \pm 0.01 \mathrm{eV}[16]{ }^{1}$

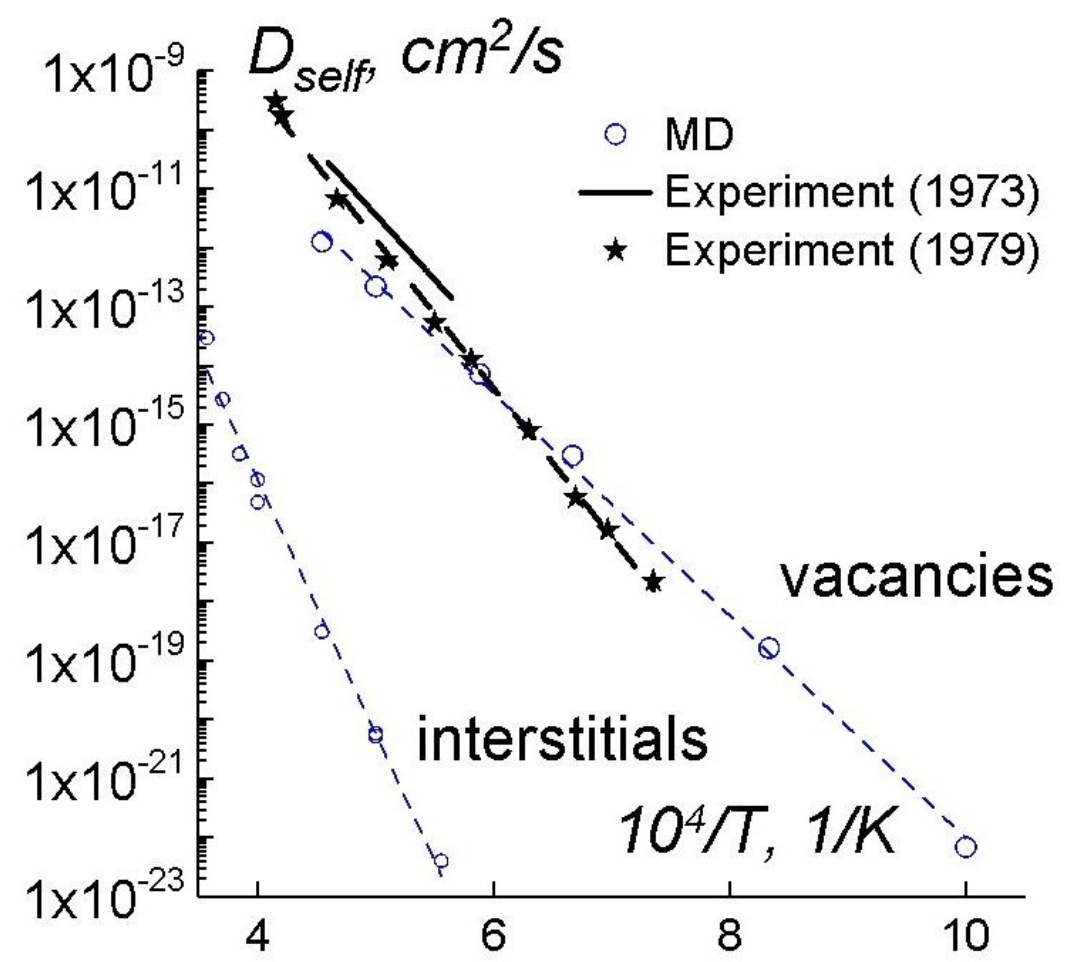

Figure 2. Calculated diffusion coefficients (dashed lines) compared to experiment $[17,18]$. The vertical axis represents the value of the diffusion coefficient and the horizontal axis $10^{4} / \mathrm{T}$.

The thermally activated mobility of a number of defects was analyzed over a wide range of temperatures. The diffusion coefficients and the frequencies of rotation of the axis of migrating crowdions were evaluated from MD simulations. The results for the calculated self-

\footnotetext{
${ }^{1}$ The accuracy of DFT simulations is $0.01 \mathrm{eV}[6]$.
} 
diffusivity of Mo atoms ( $\left.D_{\text {self }}=c_{\mathrm{v}} D_{\mathrm{v}}+c_{\mathrm{i}} D_{\mathrm{i}}\right)$ due to vacancies and interstitials (dark dashed line) are compared with experiment in Figure 2. Corresponding activation energies were estimated as $1.15 \mathrm{eV}$ for vacancies and $0.02 \mathrm{eV}$ for interstitials [16]. The calculated values for $D_{\text {self }}$ are in excellent agreement with the measured values.

The binary kinetic reactions for calculation of the rate constants for dimer formation and self-interstitial recombination with a vacancy and dislocation loop growth are described by the following:

$$
\left\{\begin{array}{l}
S I A+S I A \stackrel{i i}{\longrightarrow} d i S I A, \\
S I A+v a c \stackrel{i v}{\longrightarrow} 0, \\
S I A+(\text { Loop })_{n} \longrightarrow \text { iL } \longrightarrow(\text { Loop })_{n+1} .
\end{array}\right.
$$

\section{Dimer formation rate constant}

The rate constant of forward reaction, $\alpha_{\mathrm{ii}}$ in Eq. 3, was calculated by MD using two methods: two values of the rate constant at $300 \mathrm{~K}$ were obtained that deviate by a factor of 4 . The rate constant from our previous MD calculations, $\alpha_{\mathrm{ii}(\mathrm{MD}-1)}=4 \times 10^{13}(1 / \mathrm{s})$, was obtained by the jump method [19]; this is the largest value of the dimer formation rate constant. Using the diffusion method [19] $\alpha_{\mathrm{ii}(\mathrm{MD}-2)}=1 \times 10^{13}$ (1/s) was obtained by solving Eq. (3); this is the lowest value of the dimer formation rate. The average between these two values is $\left\langle\alpha_{\mathrm{MD}}\right\rangle=2.5 \times 10^{13}$ $(1 / \mathrm{s})$.

As discussed in Section 2, since the diffusion motion at low temperature is fully onedimensional, an additional steric factor proportional to $\exp \left(-E_{\mathrm{rot}} / k_{\mathrm{B}} T\right)$ should be included in the MD rate constants. This takes into account the activation barrier for rotation and makes possible association of two SIAs into one dimer (diSIA):

$$
\begin{aligned}
& \qquad \begin{array}{l}
i i \\
\text { where }
\end{array}{ }_{M D}>\times \times_{0} \exp \left(E_{i m} / k T\right), \\
& <{ }_{M D}>=2.510^{13}(1 / \mathrm{s}), \\
& =0.5 \exp \left(E_{\text {rot }} / \mathrm{kT}\right), \\
& E_{\text {im }}=0.02 \mathrm{eV}, \\
& E_{\text {rot }}=0.05 \mathrm{eV}, \\
& { }_{0}=5.389 .
\end{aligned}
$$

As shown in Figure 3, Eq. (4) is the rate constant for dimer formation, where the steric factor, i.e. the SIA activation energies of rotation $E_{\text {rot }}=0.05 \pm 0.01 \mathrm{eV}$ [20]. The steric factor describes the probability for two crowdions encountering each other and rotating from one equivalent direction [111] to another via a [110] state, which is a saddle point through a barrier of $E_{\text {rot }}$. 
For $E_{\mathrm{rot}}=0.05 \mathrm{eV}$, Eq. (4) gives $\alpha_{\mathrm{ii}}=4.3928 \times 10^{10}(1 / \mathrm{s})$ at $T=300 \mathrm{~K}$. The maximum and minimum values of this coefficient were obtained at $300 \mathrm{~K}$ by using two different methods (jump and diffusion method): $\alpha_{\mathrm{ii}(\max )}=7.029 \times 10^{10}(1 / \mathrm{s})$ and $\alpha_{\mathrm{ii}(\min )}=1.757 \times 10^{10}(1 / \mathrm{s})$; these values are shown by the error bars in Figure 3.

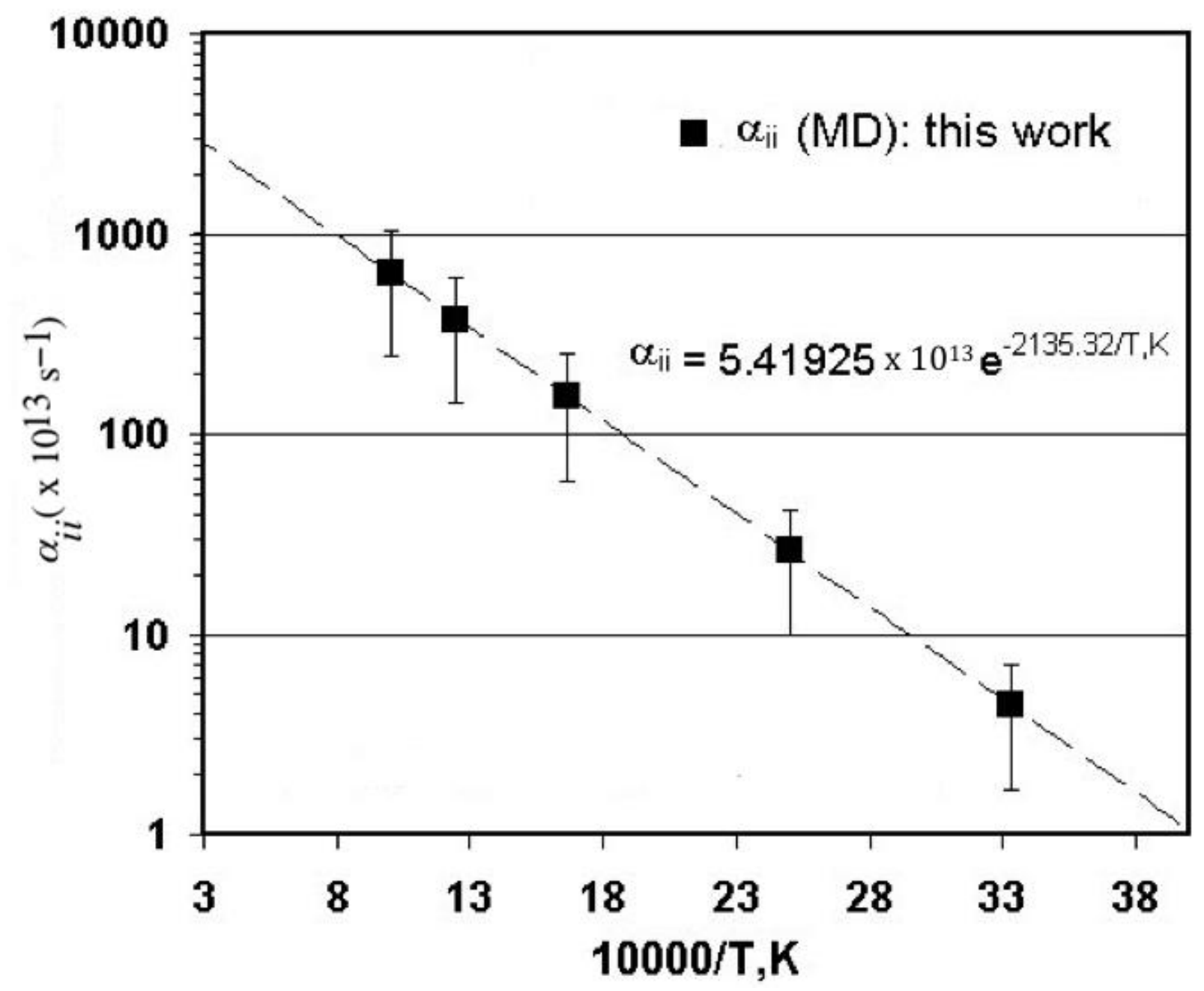

Figure 3. Dimer (diSIA) formation rate calculated using MD; see Eq. (4). The dashed line is a fit to the calculated data points.

\section{Rate constant of SIA-vacancy recombination}

The rate constant for self-interstitial recombination with a vacancy in molybdenum, ${ }_{i v}$, was calculated with MD in a previous paper [19] and is shown in Figure 4. Between 300K and $400 \mathrm{~K},{ }_{i v}$ has a constant value of $2 \times 10^{12} \mathrm{~s}^{-1}$. At temperatures above $400 \mathrm{~K}$, rises rapidly and can be represented by

$$
{ }_{i v}={ }_{i v}^{o} \exp \left(E_{a} / k_{B} T\right),
$$


where the best approximated values of the parameters were obtained by exponential fitting of the MD data and are given by ${ }^{o}=4.70235 \times 10^{13}(1 / \mathrm{s})$ and $E_{a} \approx 0.13 \mathrm{eV}$.

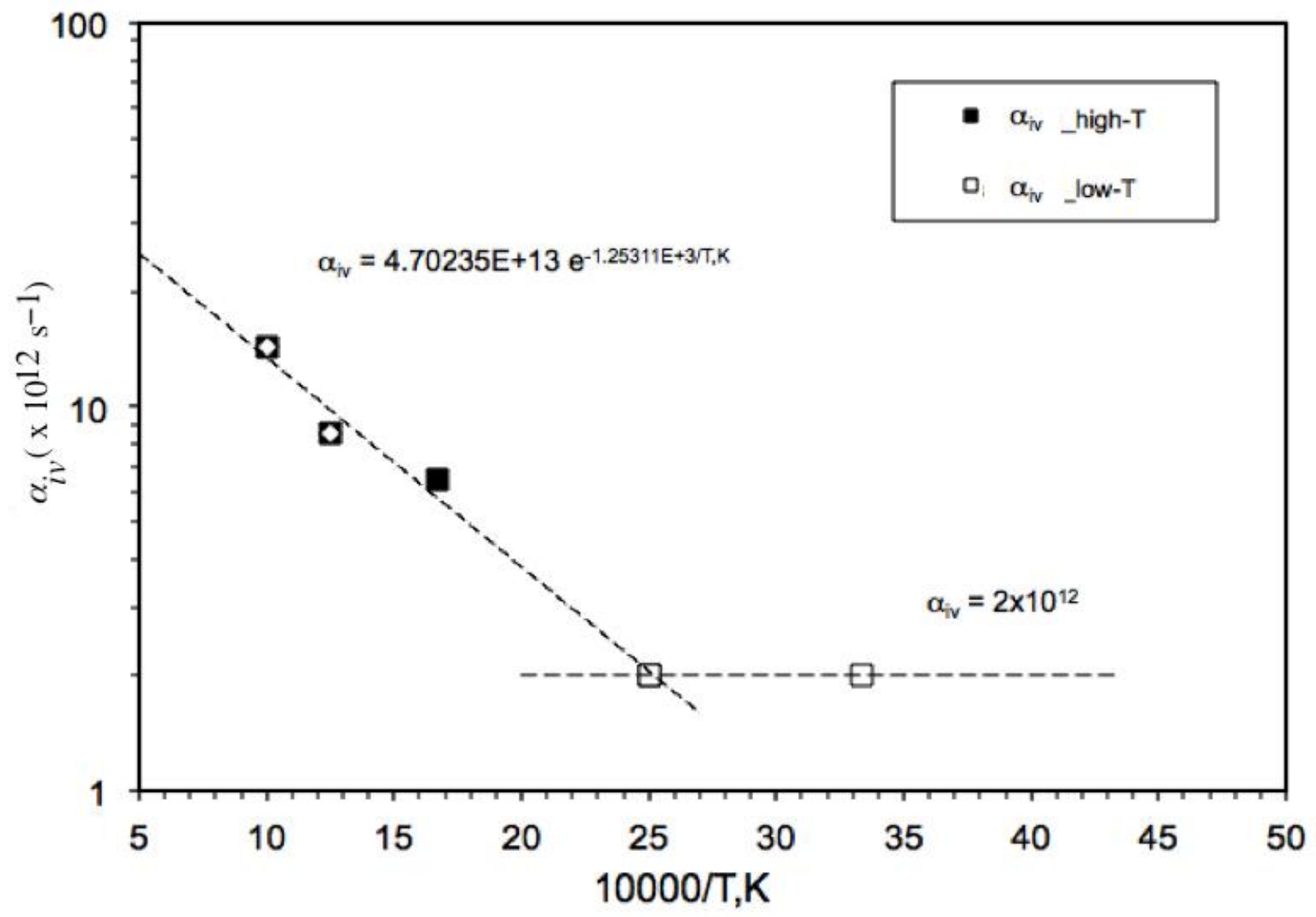

Figure 4. Self-interstitial-vacancy recombination rate in Mo calculated by using MD [19]. The dashed line is a fit to the calculated data points.

Although the MD simulations did show a very small activation barrier for recombination of an SIA with a di- and trivacancies, the results show no barrier for molecular static calculations when the reaction involves recombination of an SIA with a single vacancy. Therefore, the expression given by Eq. (5) does not contain a saddle point energy barrier factor, i.e. $\exp \left(E_{r o t} / k T\right)$.

\section{Rate constant for dislocation loop growth}

The rate constant for dislocation loop growth was obtained in this work by MD calculations of a Mo crystalline sample containing of about $2 \times 10^{6}$ Mo atoms and 56 dislocation loops, each of them built by $9,19,37$ and 61 self-interstitials, at 300K. Additionally, a different number of self-interstitials were injected into the system and the growth of the dislocation loops via SIA association mechanism of a SIA to a loop was followed by visualization of the simulated events. 
Using a small concentration of SIAs, thus reducing the frequency of dimer formation that would have altered the result, preserved the accuracy of this method. The following reaction was simulated (see Eq. 3-3):

$$
\begin{aligned}
& S I A+(\text { Loop })_{n} \longrightarrow{ }_{i L} \longrightarrow(\text { Loop })_{n+1} \\
& \frac{d c_{i}}{d t}={ }_{i L} c_{i} c_{L}, \\
& c_{i}(t)=c_{0} \exp \left({ }_{i L} c_{L} t\right),
\end{aligned}
$$

where $c_{j}(\mathrm{j}=\mathrm{i}, \mathrm{L})$ are the number concentrations of SIA and loops, and ${ }_{i L}$ is the rate constant for SIA association with loop.

The plots for the SIA concentrations were drawn as a function of time and the results for the rate constant were obtained from the tangent of the exponential fit shown in Figure 4. $9,18,37$, and 61 SIAs were placed into a crystalline MD lattice in such a way that their Burgers vector was collinear to the $<111>$ direction. The system containing 56 loops was equilibrated at $300 \mathrm{~K}$ for $100 \mathrm{ps}$. After equilibration, 25 SIAs were injected into the system and the system evolution was calculated via the number of SIAs that were identified according to symmetry and potential energy criteria [7].

Figure 5 shows MD results of the calculation where the total number of self-interstitials was introduced at the beginning of the simulation. The evolution of the system was followed for $1 \mathrm{~ns}$. Initially, the number of SIAs quickly dropped from 25 to approximately 12, mainly due to dimer formation. Therefore, the loop association coefficient was obtained for the time interval from 0.3 to $1 \mathrm{~ns}$ (red lines in Figure 5). These calculations were continued for the loops containing 9, 18, and 37 interstitials, and plots similar to Fig. 5 were drawn based on our MD calculations.

Figure 6 shows the calculated dependence of ${ }_{i L}$ on loop size. Also shown in Figure 6 is a fit to the data. The logarithmic (slow) dependence of ${ }_{i L}$ on loop size was obtained in our MD calculations and it can be explained by the existence of a certain effective reaction radius between SIAs and loops [21].

The effective radius obtained from resistivity experiments is between 0.7 and 2.5 of the lattice constant for molybdenum [22]. Therefore, the interaction energy between SIAs and loops will saturate at larger loop sizes and, as a consequence, the kinetic rate constant approaches an asymptotic value for loop sizes above 50-100 SIAs. 


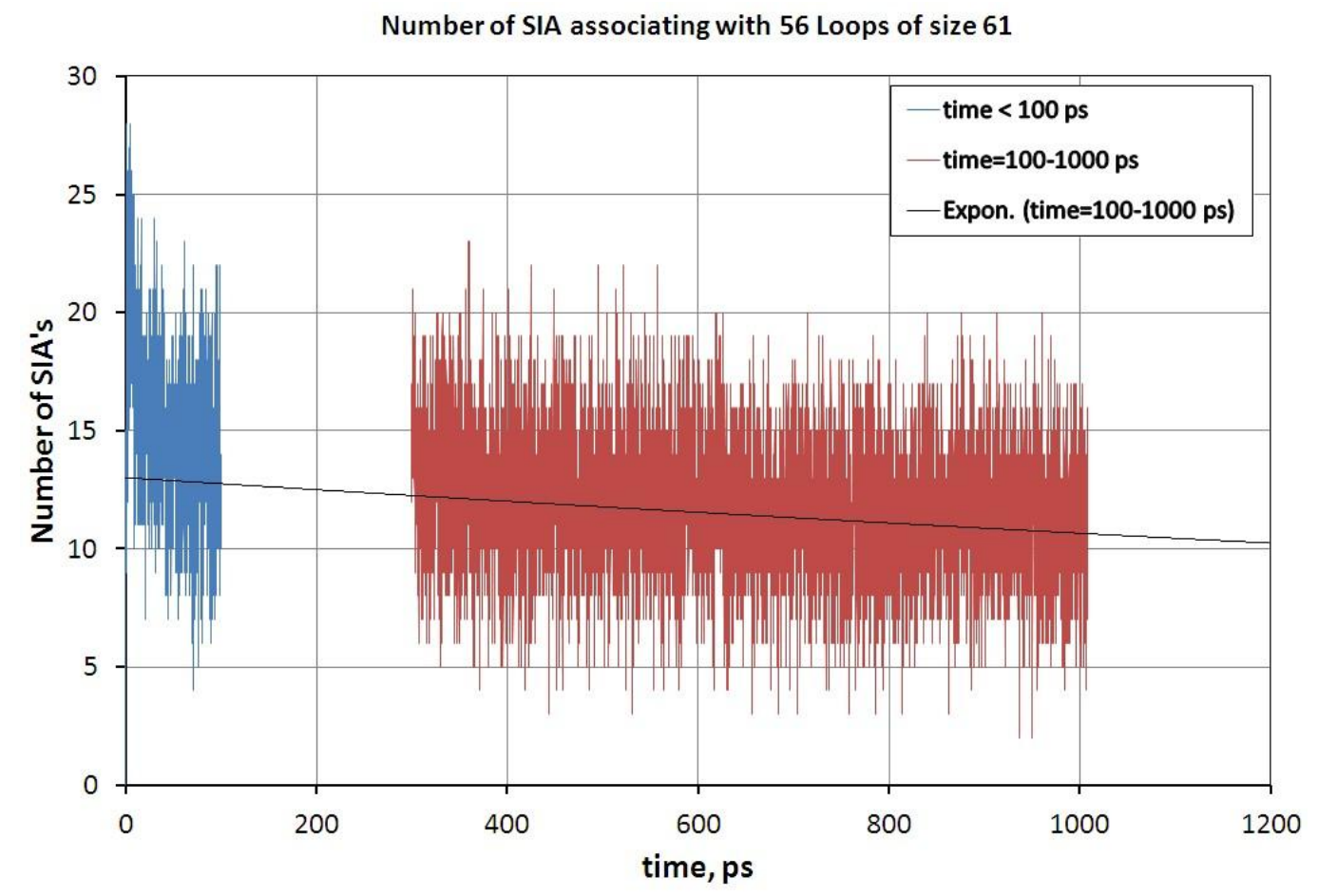

Figure 5. MD calculated results for the number of SIAs associating with dislocation loops of size 61: $n_{\mathrm{i} 0}=25, n_{\mathrm{L}}=56$.

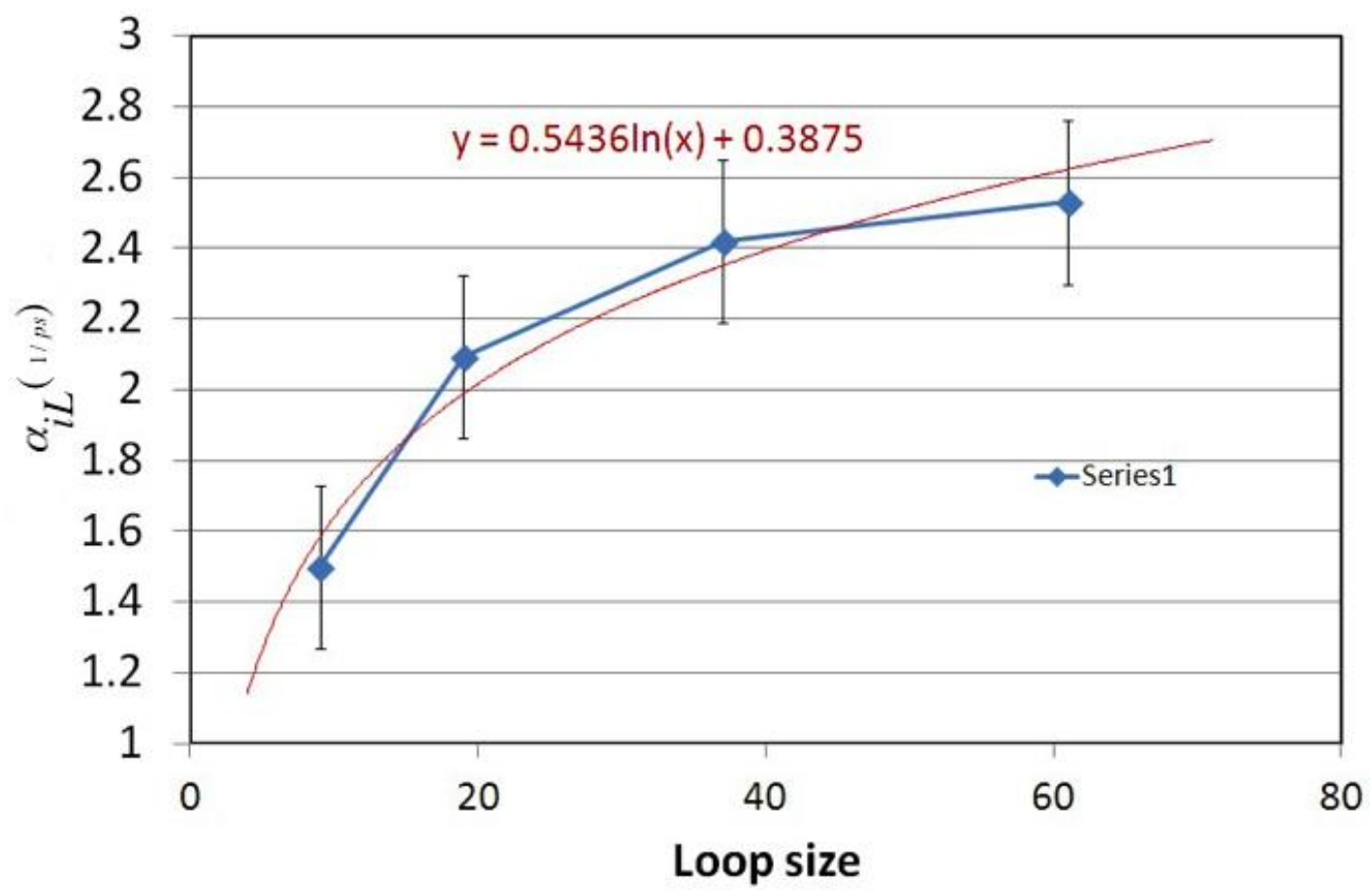

Figure 6. The kinetic rate constant for SIA-Loop association calculated by molecular dynamics in this work. Series 1 is the MD calculated data and the connecting lines are to guide the eye. 


\section{Sink strength of the surface}

The foil surface sink strengths have been derived using an embedding model and are given by [23]

$$
k_{i s}=\frac{1 / 2}{L}^{1 / 2} \operatorname{coth}\left({ }^{1 / 2} L{\frac{1}{\left({ }^{1 / 2} L\right)}}^{1}\right.
$$

where for the present case

$$
=b_{v} N_{l} m_{l} /
$$

where $b_{v}$ is the Burgers vector, the atomic volume and L the half thickness of the Mo foil. $k_{i}$ and $k_{v}$ in Eq. 1, are obtained by multiplying Eq. 9 by a factor that accounts for the boundary surface energy barrier.

Figure 7 shows the results of calculations of the energy barrier for a SIA to leave the bulk by escaping to the surface. The calculations were carried out using a new nudged elastic band (NEB) method [24-25, 27]. NEB is an atomistic method that can find the atomic configurations, the structure, and the energy barriers for the moves of the probe atom in a realistic atomic environment. The movement of the probe atom is closely correlated with the positions of the nearest neighbors. This method was implemented in LAMMPS based on the results of the work of several groups [15]. The NEB method was applied to calculations for the potential energy of an SIA moving from the bulk toward the foil surface. As demonstrated in Figure 7, there is a potential energy barrier $E_{S} \sim 0.3 \mathrm{eV}$ at the foil surface due to forces on the surface that act to minimize the surface area. As such, $k_{i}$ in Eq. 1 is given by

$$
k_{i}=\frac{1}{L}^{1 / 2} \operatorname{coth}\left({ }^{1 / 2} L{\frac{1}{\left({ }^{1 / 2} L\right)}}^{1} \exp \left(E_{s} / k T\right)\right.
$$

A similar expression can be derived for $k_{v}$.

\section{Validation of Kinetic Theory}

The multiscale model consists of the rate equations given by Eq. (1) with the MD-calculated kinetic coefficients given in Figs. 3 - 5 and Eq. (4). Two sets of experimental data [28, 29], both obtained from in situ, high-voltage electron irradiation on high-purity molybdenum, 


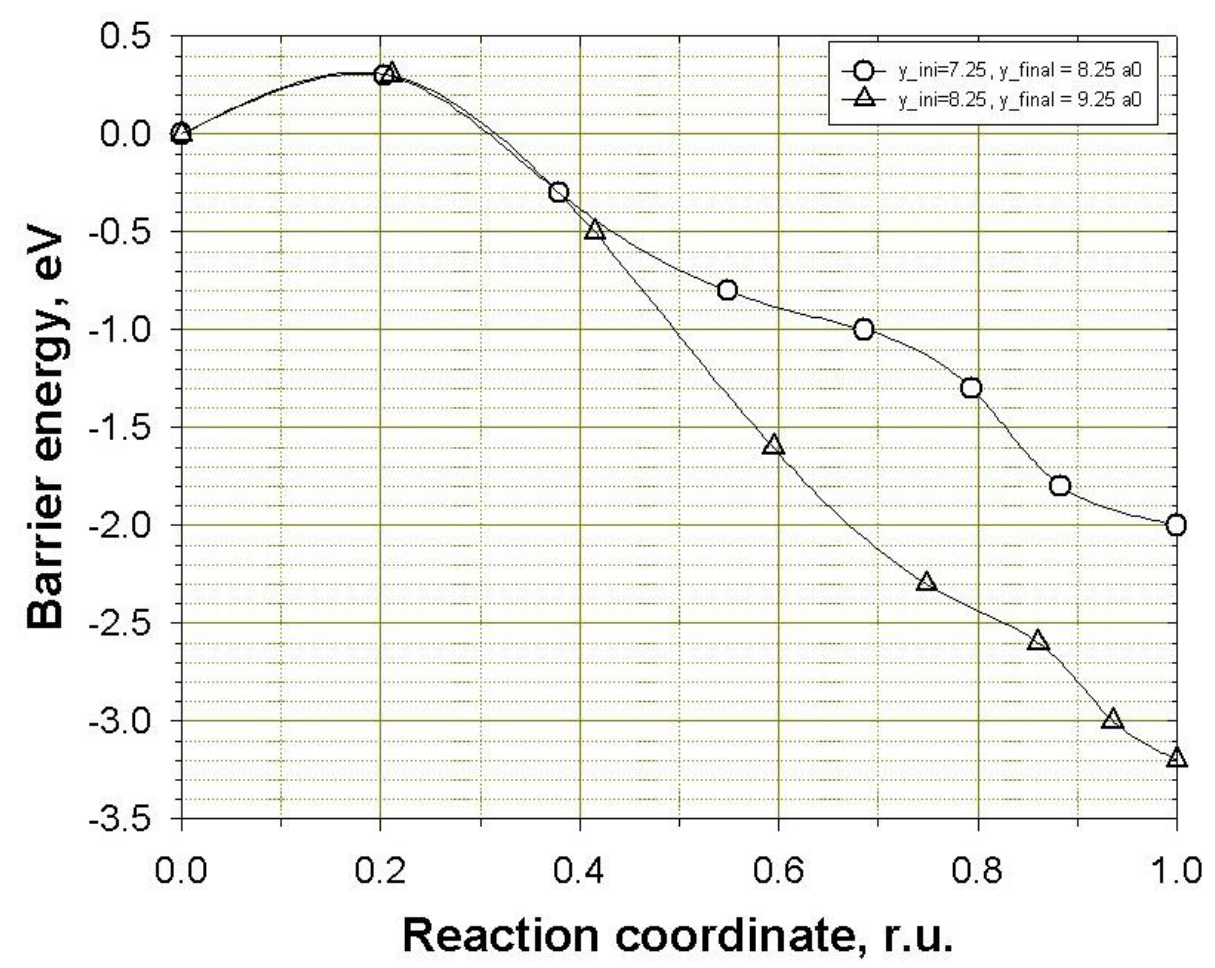

Figure 7. Calculated potential energy of an SIA as a function of distance from foil surface (0.0) for two possible migration paths.

were employed for model validation. In situ Transmission Electron Microscopy (TEM) was used in both experiments to quantitatively investigate the formation and growth of interstitial dislocation loops. The Phillipp experiment [28] was conducted at room temperature with a current density of $4.5 \times 10^{19}$ electron $/ \mathrm{cm}^{2} / \mathrm{s}$, and the Kiritani et al. experiment [29] was performed at various temperatures with a current density of $4.1 \times 10^{18} \mathrm{electron} / \mathrm{cm}^{2} / \mathrm{s}$.

Four MD-calculated physical parameters were analyzed: SIA saddle-point energy $E_{r o t}$, SIA migration enthalpy $E_{i m}$, the rate constant of recombination $\alpha_{i v}$, and the boundary surface energy $E_{S},{ }_{i i}$, and ${ }_{i L}$ are functions of $E_{r o t}$ and $E_{i m}$. Therefore, the uncertainty range of $\alpha_{i i}$ and ${ }_{i L}$ is associated with the uncertainty range of $E_{r o t}$ and $E_{i m}$, which is expressed as follows:

$\frac{i i}{i i}=\frac{i L}{i L}=\frac{\sqrt{\left(E_{r o t}\right)^{2}+\left(E_{i m}\right)^{2}}}{k T}$,

where $\Delta \alpha_{i i}$ and $\quad{ }_{i L}$ are the uncertainty ranges of $\alpha_{i i}$ and ${ }_{i L}$ respectively; $\Delta E_{r o t}$ and $\Delta E_{i m}$ are the uncertainty ranges of $E_{r o t}$ and $E_{i m}$, respectively; $k$ is the Boltzmann constant; and $T$ is the temperature. The nominal values and uncertainty ranges of these four parameters are listed in Table 2. 
Table 2. Nominal values and variation ranges of the parameters

\begin{tabular}{|c|l|l|l|}
\hline Parameter & Nominal value & Minimum & Maximum \\
\hline$E_{r o t}$ & $0.05 \mathrm{eV}$ & $0.04 \mathrm{eV}$ & $0.06 \mathrm{eV}$ \\
\hline$E_{i m}$ & $0.02 \mathrm{eV}$ & $0.01 \mathrm{eV}$ & $0.03 \mathrm{eV}$ \\
\hline$\alpha_{i v}$ & $2.0 \times 10^{12} \mathrm{~s}^{-1}$ & $1.22 \times 10^{12} \mathrm{~s}^{-1}$ & $2.78 \times 10^{12} \mathrm{~s}^{-1}$ \\
\hline$E_{S}$ & $0.2 \mathrm{eV}$ & $0.1 \mathrm{eV}$ & $0.3 \mathrm{eV}$ \\
\hline
\end{tabular}

The first part of the validation study was performed in order to evaluate the effect of key physical parameters on interstitial loop growth, and to quantify the degree of uncertainty in the calculated results. Experimental data obtained by Phillipp [28] from a real-time TEM observation of loop growth during an $1100 \mathrm{keV}$ electron irradiation at $300 \mathrm{~K}$ was utilized for this part of the study. According to this current intensity, a damage rate of $1 \times 10^{-4} \mathrm{dpa} / \mathrm{s}$ was used in the calculation. The TEM specimens were prepared from high-purity Mo rods by cutting, grinding and jet thinning. The selected observation areas were free of pre-existing extended defects, such as dislocation lines and grain boundaries. It is thus reasonable to assume that no internal sinks are in the observation areas. Other important sinks for point defects, in addition to pre-existing extended defects, are the specimen surfaces. Point defects generated by irradiation can escape to the specimen surface, which reduces the formation of defect clusters. The nominal value of the thickness of the observed areas in the experiment and used in the simulations is 400 $\mathrm{nm}[28]$.

Figure 8 shows the separate effect of each parameter listed in Table 2 on the calculated interstitial loop growth as a function of the square root of irradiation time. Also shown in Figure 8 are the experimentally measured values. In order to show the individual effect of each parameter, loop growth was calculated for each parameter at the minimum and maximum values, while keeping other parameters at their nominal value. Calculated results are displayed in Figures 8 (a), (b), (c), and (d) for $E_{r o t}, E_{i m}, \alpha_{i v}$ and $E_{S}$, respectively. The comparison shows that $E_{r o t}$ and $E_{i m}$ are the dominant parameters controlling the loop growth process, and they have a similar effect on loop size evolution (Figures 8 (a) and (b)). This is because both are embedded in the SIA-loop reaction rate constant $\alpha_{i L}$ in the form of $e^{-\frac{E}{k T}}$. Here, $E=E_{r o t}$ or $E_{i m}$. Compared with $E_{r o t}$ and $E_{i m}, \alpha_{i v}$ and $E_{S}$ have less influence on the results, since the loop size does not change as much within their uncertainty ranges (Figures $8(\mathrm{c})$ and (d)). Note that the calculated loop-size evolution, shown in Figure 8, obeys a linear relationship with the square root of time, which is consistent with the experimental observation (Figure 2 in [28]). Based on the qualitative agreement between key loop growth characteristics and the experimental data, we conclude that this multiscale model can reasonably describe defect evolution during electron irradiation at room temperature.

Figure 9 shows the upper and lower limit of the calculated evolution of dislocation density vs. time using the range of parameter values listed in Table 2 . The calculated dislocation loop density bounds the range of the measured density $\left(\approx 10^{14} \mathrm{~cm}^{-3}\right)$ [28]. In addition, the trend 
of calculated density follows the observed relatively constant loop density as a function of irradiation time.
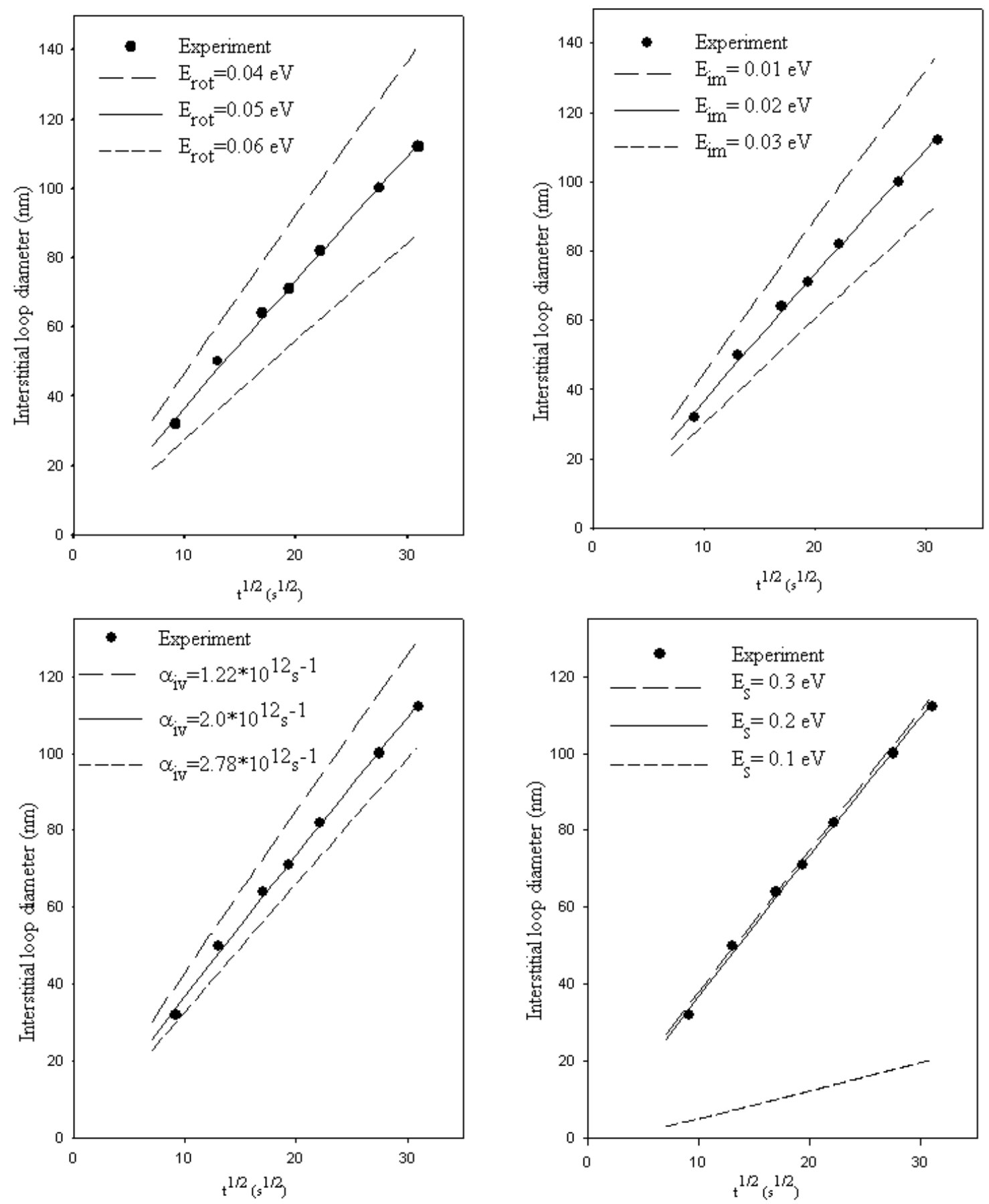

Figure 8. Effect of various parameters on calculated dislocation loop size (a) SIA activation energy of rotation $E_{r o t}$, (b) SIA migration enthalpy $E_{i m}$, (c) the rate constant of recombination $\alpha_{i v}$, and (d) the surface barrier energy $E_{S}$. The experimental data are from Ref. [28] 


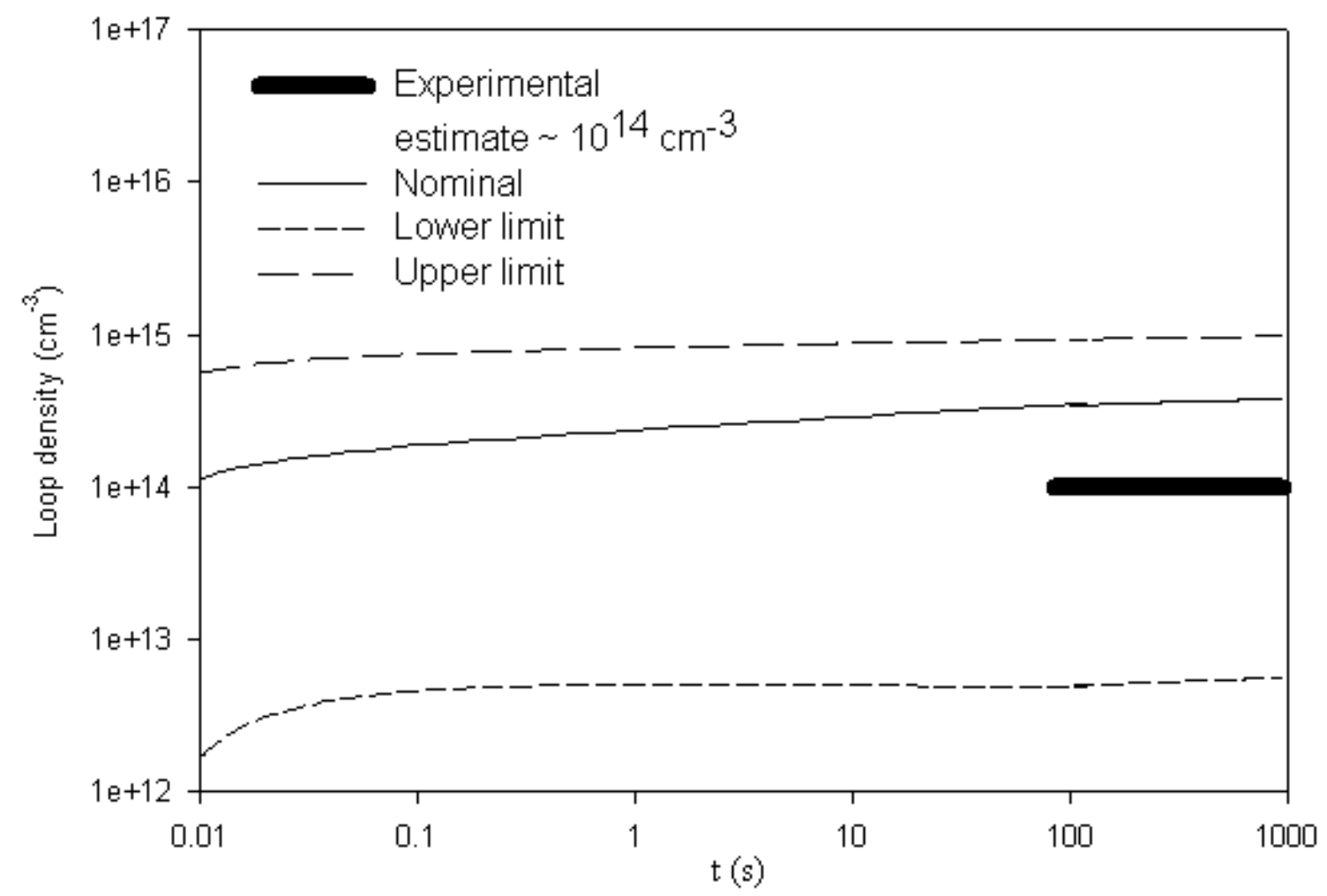

Figure 9. Evolution of simulated dislocation loop density over time compared to data estimated from Phillip's experimental observation (with lower and upper limit to show the calculated uncertainty) [28].

The second part of the model validation uses the experimental data of Kiritani et al. [29]. TEM specimens used in this experiment were also prepared from high-purity polycrystalline Mo plates. They were electrolytically thinned to have a smoothly tapered specimen edge where quantitative experiments could be performed. In situ irradiation was carried out with $1500 \mathrm{keV}$ electrons under a constant flux of $4.1 \times 10^{18}$ electron $/ \mathrm{cm}^{2} \mathrm{~s}$. According to this current intensity, a damage rate of $1.23 \times 10^{-4} \mathrm{dpa} / \mathrm{s}$ was used in the calculation. Temperature dependence of the loop formation was studied by counting loop densities from TEM micrographs obtained at various irradiation temperatures, from $25^{\circ} \mathrm{C}$ up to $400^{\circ} \mathrm{C}$ (Figure 8 in [29]).

Figure 10 shows the evolution of simulated dislocation loop density as a function of irradiation time for two different irradiation temperatures compared to data estimated from experimental observation in [29]. The calculated results show that the interstitial loop densities gradually increase with irradiation time at both $25^{\circ} \mathrm{C}$ and $200^{\circ} \mathrm{C}$, and the loop density at $25^{\circ} \mathrm{C}$ is higher than that at $200^{\circ} \mathrm{C}$. The reason for the decrease in density with increased temperature is due, in part, to the increased loss of SIA' a to the specimen surface. The calculated results follow the general trend observed in the experiment. 


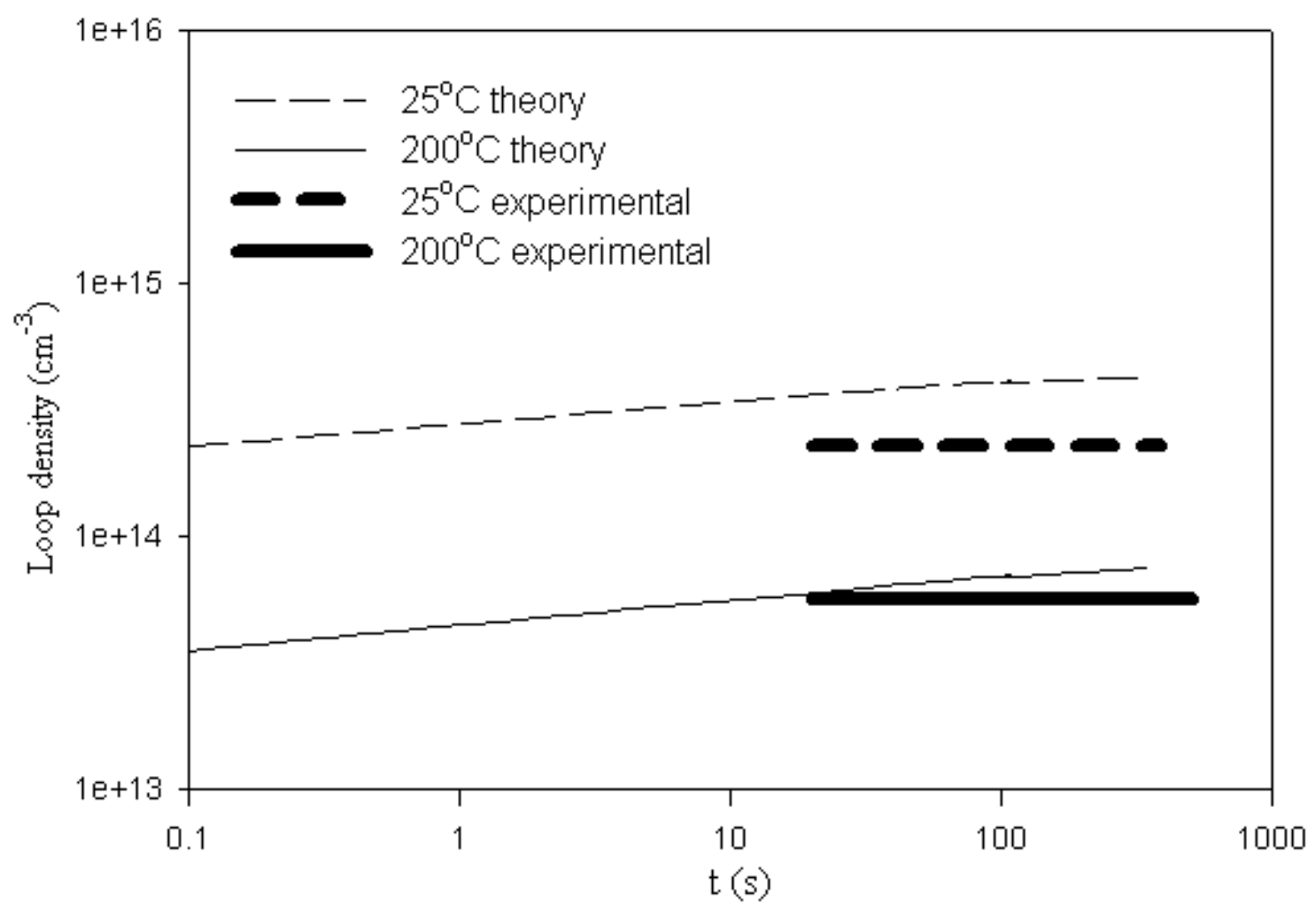

Figure 10. Evolution of simulated dislocation loop density vs. time compared with data estimated from experimental observation in [29].

\section{Discussion and Conclusion}

The kinetic rate-theory constant ( ${ }_{i L}$ in Eq. 1) for interaction between SIA's and loops was derived in Section 7 using MD. The resulting expression (see Fig. 6) is very weakly dependent on the loop size, and is a consequence of the predicted 1-D diffusion of SIA's operative at the relatively low temperatures of the electron irradiation experiments being studied in this work. The observed linear behavior of the loop size versus the square root of the irradiation time demonstrated in Figure 8 is directly explained by this newly derived expression. In contrast, the mean-field expression for the kinetic rate constant of SIA association to a loop can be expressed as follows [30]:

$$
{ }_{i L}=2 \quad Z_{i} D_{i} \sqrt{N_{l} A /} /,
$$

where $Z_{i}$ is the bias for interstitials, the atomic volume, and $A$ the area per atom. Eq. 11 was derived by assuming 3-D diffusion of SIA's and solving the diffusion to an effective sphere. The use of the relatively strong square root dependence of ${ }_{i L}$ on $N_{l}$ given by Eq. 11 in Eq. 1 results in non-linear behavior of the loop growth as a function of the square root of irradiation time, in direct disagreement with the observations shown in Figure 8. 
Often, rate theory comparisons with data are obtained by adjusting ("predicting" unknown critical parameters in order to obtain optimal agreement with the data. In principle, these "predicted" physical parameters and materials properties can be obtained experimentally, but these measurements are rarely accomplished because of the expense and difficulty involved in dealing with irradiated materials. The uncertainty caused by the presence of these unverified materials properties casts a shadow on the validity of the underlying physics used to build the mathematical model for the phenomena.

For example, in addition to the incorrect shape of the loop growth vs. square root of irradiation time discussed above, if one were to apply the rate theory given by Eq. (1) in this manner, the SIA diffusion coefficient "predicted" by comparison of calculated loop diameter and density with data would be many times smaller than that calculated by MD. The nominal value of the MD calculated SIA migration enthalpy as shown in Table 2 is $0.02 \mathrm{eV}$. Typical migration enthalpies utilized in typical rate theory calculations of defect behavior in irradiated metals are several tenths to about $1 \mathrm{eV}$. A rate theory model employed in this manner would have the incorrect physics, e.g. SIA's having to surmount the $<110>$ saddle point energy barrier in order to form dimers and to interact with interstitial loops. It is the combination of very fast 1-D diffusion in the <111> direction and the saddle point energy barrier that results in expressions for ${ }_{i i}$ and ${ }_{i L}$ kinetic coefficients that are validated against experimental data and that result in a predictive instead of interpretative model.

The multiscale method presented in this paper, i.e. using molecular dynamics to obtain parameters for other long scale models, such as rate theory, has been used at least since the early 90's for the case of nuclear materials [31-32]. More recent work has shown how the coupling between MD results and rate theory can give the wrong answer in some situations due to the lack of spatial correlations between defects in a mean field approach [33]

Inherent in the multiscale model is the assumption that materials properties and kinetic coefficients calculated by ab initio and molecular dynamics methods in the nanometer and nanosecond time scales are valid in the micron and second scales. In addition, rate theory is generally based on continuum theory, which smooth's out variations in defect fluxes over mesoscale distances. Although kinetic Monte Carlo studies have demonstrated the accuracy of the mean field theory approach in describing atomistic processes (e.g., 3D normal grain growth) [30], it is not a priori obvious that this approximation is valid for the type of defect kinetics explored here. The successful implementation of the multiscale analysis presented in this work provides a measure of the validity for mapping of length and time scales, as well as for the continuum approximation.

The model described by Eq. 1 only includes sessile loops (e.g. ignores the possibility of loop diffusion to the specimen surface as well as loop-loop interaction), and does not take into account larger defect clusters and their migration and interaction. However, it is the opinion of the authors that the inclusion of these effects would not alter the basic conclusions of this work.

This paper differs from previous work (e.g. see Ref. 34-37) in that the objective of the analysis is not the optimization of various model parameters and materials properties, but is on the use of MD to provide values for uncertain materials properties and kinetic coefficients for 
use in rate theory calculations on the irradiation behavior of metals and metal alloys. The only parameter that has not been calculated is the SIA activation energy of rotation $E_{\text {rot. }}$ Based on comparison with the irradiated data, the value of $E_{\mathrm{rot}}$ was predicted to have a value of $\approx 0.05 \mathrm{eV}$. This value is consistent with that used for the analysis of defect behavior in iron [20].

The successful implementation of a multiscale approach of combining ab initio/molecular dynamics and rate theory modeling described in this paper demonstrates the viability of using MD methodology to improve our understanding of the underlying physics in complex irradiation environments (e.g., with separate effects MD simulations) and of conducting future efforts aimed at developing a predictive performance model of material behavior in irradiation environments.

\section{ACKNOWLEDGMENTS}

The Authors thank Drs. A.V. Yanilkin, A.Yu. Kuksin, V.V. Stegailov and P. A. Zhilyaev. (Joint Institute for High Temperatures, Moscow, Russian Federation) for many valuable discussions, and S. Nurkenov for his assistance with the rate theory calculations. 


\section{REFERENCES}

[1] J. Rest, "Modeling of Fission-Gas-Induced Swelling of Nuclear Fuels," Rudy Konings, ed., Comprehensive Nuclear Materials, Elsevier Science ISBN: 978-0-08-056027-4, Chapter 3.20, March 2012, Pages 579-627.

[2] G. Kaganas and J. Rest, "A Physical Description of Fission Product Behavior in Fuels for Advanced Power Reactors," Argonne National Laboratory Report ANL-07/24 (2007), DOI 10.2172/919331, http://www.osti.gov/scitech/biblio/919331.

[3] S.V. Starikov, Z. Insepov, J. Rest, A.Y. Kuksin, G.E. Norman, V.V. Stegailov, A.V. Yanilkin, Phys. Rev. B 84 (10), 104109 (2011).

[4] S. Han, L. A. Zepeda-Ruiz, G. J. Ackland, R. Car, D. J. Srolovitz, Phys. Rev. B 66, 220101 (2002).

[5] D. Nguyen-Manh, A. P. Horsfield, S. L. Dudarev, Self-interstitial atom defects in BCC transition metals: Group-specific trends, Phys. Rev. B 73, 020101(R) (2006).

[6] P. Ehrhart, P. Jung, H. Shultz, H. Ullmaier, Atomic Defects in Metals, ed. by H. Ullmaier Landolt-Bornstein New Series, Group III, Vol. 25 (Springer-Verlag, Berlin, 1991).

[7] R. Pasianot, M. Alurralde, A. Almazouzi, and M. Victoria, Philos. Mag. A 82, 1671 (2002).

[8] M. W. Finnis and J. E. Sinclair, Philos. Mag. A 50, 45 (1984).

[9] P. M. Derlet, D. Nguyen-Manh, S. L. Dudarev, Phys. Rev. B 76 (5) (2007) 054107.

[10] F. Ercolessi, J.B. Adams, Europhys. Lett. 26 (1994) 583.

[11] P. Brommer, F. Gähler, Modelling Simul. Mater. Sci. Eng. 15(2007) 295-304.

[12] G. Kresse, J. Furthmuller, Phys. Rev. B 54 (1996) 11169.

[13] S.P. Fitzgerald, D. Nguyen-Manh, Phys. Rev. Lett. 101 (2008) 115504.

[14] Z. Insepov, J. Rest, A.M. Yacout, A.Yu. Kuksin, G.E. Norman, V.V. Stegailov, S.V.

Starikov, A.V. Yanilkin, Journal of Nuclear Materials 425 (2012) 41-47.

[15] S. J. Plimpton, J. Comp. Phys. 117 (1995) 1-19.

[16] Zeke Insepov, Jeffrey Rest, Abdellatif M. Yacout, Bei Ye, Di Yun, Alexey Y Kuksin, Genri

E Norman, Vladimir V Stegailov and Alexey V Yanilkin (2012). Atomistic and Kinetic

Simulations of Radiation Damage in Molybdenum. MRS Proceedings, 1444, mrss12-1444-s08-

04 doi:10.1557/opl.2012.1478.

[17] G.B. Federov, E.A. Smirnov, V.N. Gusev, F.I. Zhomov, V.L. Gorbenko, in Metallurgy and Materials Science of Pure Metals, Atomizdat, Moscow, vol. 10 (1973) 62-68.

[18] K. Maier, H. Mehrer, and G. Rein, Zeitschrift fuer Metallkunde, 70 (1979) 271.

[19] A. Yanilkin, Z. Insepov, G. Norman, J. Rest, V. Stegailov, Atomistic simulation of clustering and annihilation of point defects in Molybdenum, 2012, Defect and Diffusion Forum, 323-325, 95 .

[20] Ming Wen, Akiyuki Takahashi, Nasr M. Ghoniem, , J. Nucl. Mater. 392 (2009) 386-395.

[21] U. M Gösele, Reaction kinetics and diffusion in condensed matter, Prog. Reaction Kinetics, 13, (1984) 63-161.

[22] H.B. Afman, Phys., State Solids A11 (1972) 705-712.

[23] R. Bullough, M.R. Hayns, and M.H. Wood, J. Nucl. Mater. 90 (1980) 44-59.

[24] Henkelman and Jonsson, J Chem Phys, 113, 9978-9985 (2000).

[25] Henkelman, Uberuaga, Jonsson, J Chem Phys, 113, 9901-9904 (2000).

[26] J. Rest, G. L. Hofman, J. Nucl. Mat. 277 (2000) 231-238.

[27] Nakano, Comp Phys Comm, 178, 280-289 (2008).

[28] F. Phillipp, Phys. Stat. Sol. (a) 104, (1987) 329-342. 
[29] M. Kiritani, Y. Maehara, H. Takata, J. Phys. Soc. Japan, 41, No. 6 (1975) 1677-1686.

[30] M. R. Hayns, , J. Nucl. Mater. 56 (1975) 267-274.

[31] R. S. Hixson, D. A. Boness, J.W. Shaner, and J. A. Moriarty, Phys. Rev. Lett. 62, 637 (1989).

[32] J. A. Elliot, "Novel approaches to multiscale modeling in materials science," International Materials Reviews, Volume: 56 Issue: 4, Pages: 207-225.

[33] C.S. Becquart and C. Domain, "Modeling Microstructure and Irradiation Effects," Metallurgical and Materials Transactions, Volume: 42A, Pages: 852-870.

[34] A. Barbu. C.S. Becquart. J L, Bocquet, J, Dalla Torre, and C. Domain: Phil. Mag., 2005, vol. 85, pp. 541-47.

[35] D. Xu, B.D. Wirth, M. Li, M.A. Kirk, Current Opinion in Solid State Materials Science, volume 16 (2012) 109-114.

[36] D. Xu, B.D. Wirth, Meimei Li, M.A. Kirk, Acta Materialia, volume 60 (2012) 4286-4302.

[37] Meimei Li, M.A Kirk, P.M. Baldo, D. Xu, B.D. Wirth, Philosophical Magazine, volume 92 (no. 16) (2012) 2048-2078. 
Graphical Abstract
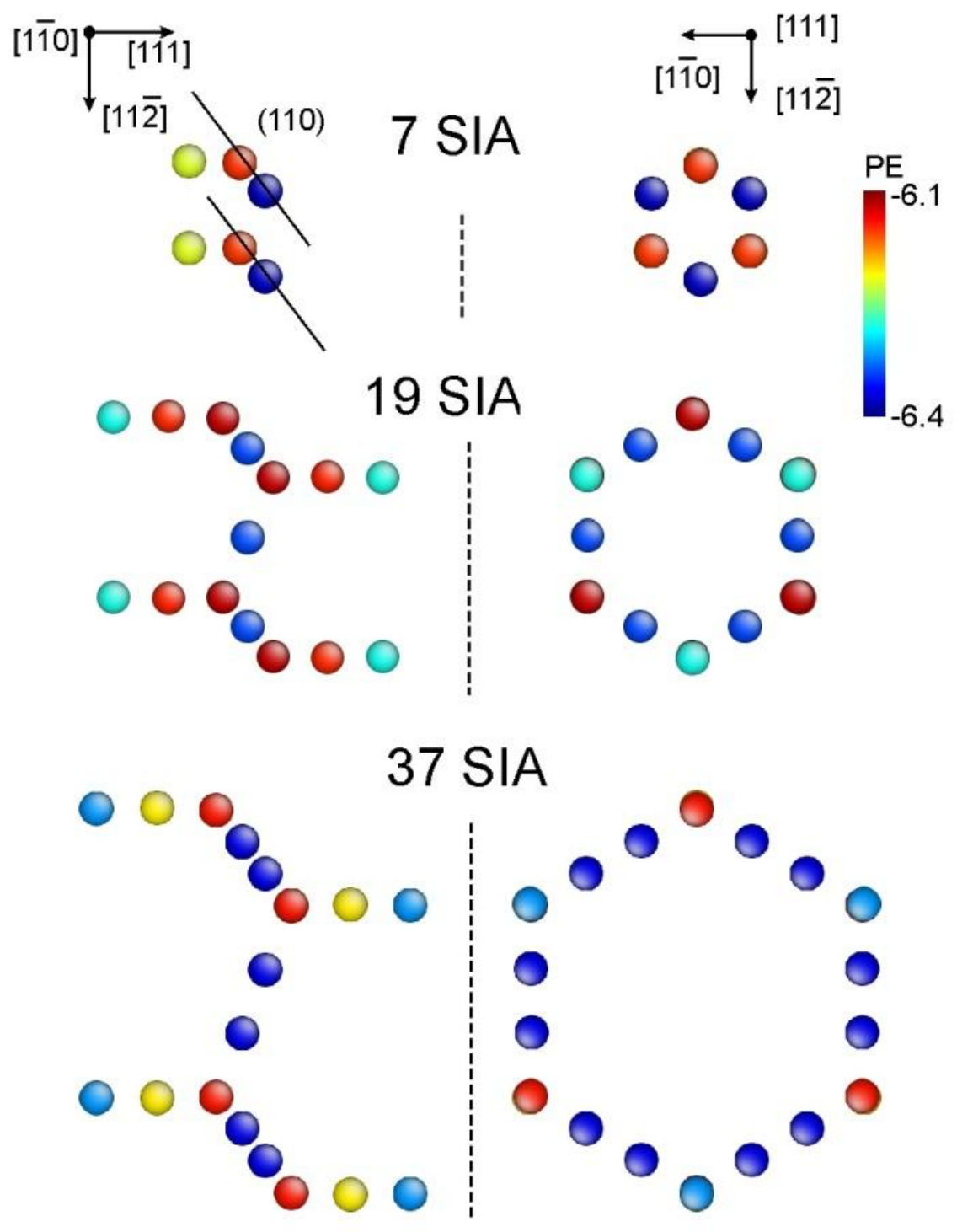

Optimized configuration of 7-SIA, 19-SIA, and 37-SIA clusters. The atoms are colored by the potential energy (PE). Only atoms with $\mathrm{PE}>-6.4 \mathrm{eV}$ are shown. 